Revista Brasileira de Cartografia

ISSN 1808-0936 | https://doi.org/10.14393/revbrascartogr

Sociedade Brasileira de Cartografia, Geodésia, Fotogrametria e Sensoriamento Remoto

\title{
Geração de Funcionais da Gravidade a partir de Observações Aerogravimétricas e de Modelos Geodésicos Globais
}

\author{
Generation of Gravity Functionalities from Aerogravimetry Observations and Global \\ Geodetic Models
}

Andrea Galudht Santacruz Jaramillo ${ }^{1}$ e Sílvio Rogério Correia de Freitas ${ }^{2}$

1 Universidade Federal do Paraná, Curso de Pós-Graduação em Ciências Geodésicas, Curitiba, Brasil. andrea.santacruz@ufpr.br ORCID: http://orcid.org/0000-0002-6648-9879

2 Universidade Federal do Paraná, Curso de Pós-Graduação em Ciências Geodésicas, Curitiba, Brasil. sfreitas@ufpr.br ORCID: https://orcid.org/0000-0003-0830-6541

Recebido: 10.2020 | Aceito: 12.2020

Resumo: A Rede Vertical de Referência do Brasil - RVRB é estruturada em dois segmentos distintos com discrepância entre si (offset). Cada qual é vinculado a um Datum Vertical - DV distinto: Imbituba e Santana. Sobre a realização 2018 da RVRB já existem determinações do offset no espaço do geopotencial com base em gravimetria e nivelamento. Estas soluções têm como pontos fracos a utilização de levantamentos sobre ramais abertos e DVs locais. A visão atual é a de que cada segmento deva ter seu offset referido a uma mesma referência global. Para tanto, é recomendada tal determinação com base em soluções do Problema do Valor de Contorno da Geodesia em referencial global. Para estas soluções são necessárias funcionais da gravidade (e.g. distúrbio da gravidade) independentes de reduções em referencias locais. Na região de conexão dos segmentos, nos estados do Pará e do Amapá, se dispõe de um extenso levantamento aerogravimétrico para finalidades geofísicas, devendo receber profunda depuração para aplicações em Geodesia. No presente trabalho são apresentadas todas as atividades de depuração e compatibilização com referencial global deste levantamento aerogravimétrico. O Modelo Digital de Altitude MERIT substituiu ao SRTM nas reduções da gravidade. O Modelo Global do Geopotencial XGM2019 em substituição ao EGM96 e a modelagem das marés permanentes, foram requisitos fundamentais para o vínculo ao espaço do geopotencial e a um único referencial global. Com as estratégias empregadas, foi obtida uma melhora significativa na aderência das funcionais geradas com modelos globais da Geodesia (RMS melhor que $7 \mathrm{mGal}$ ).

Palavras-chave: Rede Vertical de Referência do Brasil. Aerogravimetria. Compatibilização de SGRs e Sistemas de Marés Permanentes. MERIT. XGM2019.

\begin{abstract}
The Brazilian Vertical Reference Frame - BVRF is structured in two different segments with offsets between them. Each one is linked to a different local Vertical Datum - VD: Imbituba and Santana. There are, already, some determinations about the referred offset in the geopotential space related to BVRF last realization in 2018, in general, based in gravimetry and leveling associated with GPS positioning. These solutions have as limitations the use of surveys on available open leveling lines and local VDs. The current view is that each segment should have its offset referred to a same global reference surface. Such determination must be based on solutions of the Geodetic Boundary Value Problem. For this kind of solution is mandatory gravity functionals (for example, gravity disturbances) independent of reductions in local reference systems. In the connection region (in the states of Pará and Amapá), an extensive aerogravimetric survey for geophysical purposes is available. It should receive deep depuration for applications in Geodesy. This work presents the depuration activities and compatibilization with a global reference system on the aerogravimetric survey. The DEM MERIT replaced the SRTM in gravity reductions and the GGM XGM 2019 replaced the EGM96. It was introduced the modeling of the permanent tides. These actions were done aiming to accomplish the fundamental requirements related to data base for the linking BVRF segments to an only global reference. With employed strategies, a significant improvement in the adherence of gravity functionals with global geodetic models was obtained (RMS better than $7 \mathrm{mGal}$ ).
\end{abstract}

Keywords: Brazilian Vertical Reference Frame. Aerogravimetry. GRS Compatibility and Permanent Tide Systems. MERIT. XGM2019.

\section{INTRODUÇÃO}

Visando atender às demandas globais em termos da infraestrutura geodésica necessária ao 
monitoramento do Sistema Terra, a Assembleia Geral das Nações Unidas (UN, 2015), com a Resolução A/RES/69/266, estabeleceu o Global Geodetic Reference System - GGRS. Este visa atender às novas exigências em termos de Sistemas Geodésicos de Referência - SGRs globais, que associem o necessário referenciamento de processos planetários ao espaço geométrico, bem como ao espaço do geopotencial, considerando os aspectos da rotação/orientação da Terra relativamente ao espaço exterior (IAG, 2016). A realização (Frame) do GGRS (o GGRF) pressupõe envolver: 1) o referenciamento geométrico, com base no International Terrestrial Reference System - ITRS e suas realizações (Frames) ITRFyyyy, onde yyyy denota a época de referência (IERS, 2020); 2) o referenciamento ao espaço do geopotencial deve ser cumprido pelos preceitos do International Height Reference System - IHRS, com a sua respectiva realização (Frame - IHRF), conforme estabelecido pela Resolução \#1 de 2015 da International Association of Geodesy - IAG (IAG, 2015a); e 3) os aspectos da dinâmica planetária e sua rotação no espaço externo de referência, que são realizados pelos Parâmetros de Orientação da Terra, mantidos pelo International Earth Orientation and Reference Systems Service - IERS (IERS, 2020).

Com os preceitos estabelecidos desde 2003 e aprimorados até a atualidade pelo Global Geodetic Observing System - GGOS da IAG, o Instituto Brasileiro de Geografia e Estatística - IBGE estabeleceu um projeto de modernização da Rede Vertical de Referência do Brasil - RVRB. Em 2018, sua realização foi efetivada no espaço do geopotencial dotando as altitudes ajustadas de significado físico, característica até então não existente nas realizações anteriores, tal como na Rede Altimétrica de Alta Precisão - RAAP, e realizada em termos de altitudes normais-ortométricas (IBGE, 2018).

Ainda persistem na RVRB aspectos a serem superados para obter seu vínculo a um sistema global de altitudes, bem como a unificação de seus dois segmentos. Cada segmento é ainda vinculado a um distinto Datum Vertical - DV, devido a dificuldades de conexão convencional na região Amazônica: o de Imbituba SC (DV-I) envolvendo a porção majoritária da RVRB e; a porção no Estado do Amapá vinculada ao marégrafo de Santana (DV-S). Ainda, ambos os segmentos são vinculados a distintos DVs locais. Existe uma discrepância (offset) entre eles, a qual já foi estimada no espaço do geopotencial, porém, com base em linhas de nivelamento abertas, associadas com gravimetria em um único ponto de conexão e aplicação de modelos quase-geoidais de forma relativa como elementos de controle (SANTACRUZ-JARAMILLO; DE FREITAS; LUZ, 2019). No entanto, problemas ainda persistem na RVRB, como a conexão, que é baseada somente em dois ramais de nivelamento abertos sem controle de fechamento, um de Altamira no Pará até Laranjal do Jari no Amapá, e outro de Santana no Amapá até Laranjal do Jari (Figura 1). Ambos os segmentos são referidos a DVs locais vinculados à observação do Nível Médio do Mar - NMM em épocas e locais distintos. Na presente forma, as funcionais do geopotencial geradas com base nas altitudes da RVRB, têm apenas caráter local e são inconsistentes entre si e, também com referenciais globais, tal como acontece com as anomalias da gravidade utilizadas para diversas aplicações. Se observados os pressupostos atuais da IAG (IHDE; SÁNCHEZ; BARZAGHI, 2017; BARZAGHI; DE GAETANI; BETTI, 2020), a substituição dos DVs locais por um único DV global implica na solução do problema do offset entre os segmentos e os vincula ao IHRS/IHRF, tornando a RVRB consistente com todas as redes verticais globais realizadas em consonância com os mesmos critérios.

Dentre as estratégias usuais para conexão de Redes Verticais - RVs, tais como as descritas por Lehmann (2000), a forma ideal para conexão é aquela que não faça uso de funcionais baseadas em referenciais e reduções locais, dependentes somente de observações restritas a linhas ou pontos de conexão. Assim aparecem como adequadas as soluções do Problema do Valor de Contorno da Geodesia - PVCG que envolvam observações globais e regionais de duas formas. A primeira, a forma escalar livre baseada em anomalias da gravidade de Molodensky, que utiliza observações da gravidade referidas à Superfície Física da Terra - SF, devidamente posicionadas em referencial global e valores teóricos da gravidade obtidos sem hipóteses simplificativas no denominado teluroide. A segunda forma, a fixada na SF, é baseada na Integral de Hotine, que utiliza como funcionais os distúrbios da gravidade. Esta advém da diferença da gravidade real e da gravidade teórica no mesmo ponto, portanto, se considerada na superfície física, tais funcionais são facilmente obtidas em referencial global e sem reduções locais com base em hipóteses simplificativas. As soluções do PVCG mencionadas são usualmente obtidas com base em decomposição espectral das funcionais, visando à geração de componentes residuais. São necessárias observações regionais com distribuição espacial adequada, assim como a utilização de Modelos Globais do Geopotencial - MGGs para modelagem de longos e médios 
comprimentos de onda e, Modelos Digitais de Altitude - MDAs para a modelagem dos efeitos de curto comprimento de onda relacionados com a topografia regional no entorno dos pontos de cálculo.

Na região de conexão dos segmentos, conforme a Figura 1, se dispõe como única alternativa de observações gravimétricas, com distribuição regional apta ao estabelecimento de soluções do PVCG, aquelas advindas de extenso levantamento aerogravimétrico (aproximadamente oito milhões de pontos) para finalidades geofísicas, realizado em 2006 sob os auspícios da Agência Nacional do Petróleo, Gás Natural e Biocombustíveis - ANP. Sobre este levantamento aerogravimétrico já foi realizado um estudo para avaliar sua potencialidade para fins geodésicos (JESUS; DE FREITAS, 2020). Nesta avaliação detectou-se certas incompatibilidades geradas pelos SGRs envolvidos e superfícies de redução utilizadas na determinação das funcionais derivadas dos dados do levantamento, envolvendo erros em altitude na ordem de $25 \mathrm{~m}$ ou mais. Funcionais originais apresentavam discrepâncias acentuadas relativamente a observações esparsas disponíveis na região. Assim é que se optou por novos cálculos com base em dados dos sensores gravimétricos e posicionais embarcados, e a partir destes efetivar-se todas as compatibilizações de referenciais e de sistemas de marés permanentes (TENZER et al. 2011), bem como reduções e interpolações necessárias para obtenção das funcionais adequadas para a solução do PVCG nas formas mencionadas. Assim é que foram concentrados os esforços no referenciamento de todas as observações no GRS80 (TORGE; MÜLLER, 2012), na obtenção de altitudes físicas dos pontos de observação com auxílio do MGG XGM2019 (ZINGERLE et al. 2019) e do MDA Multi-Error-Removed Improved-Terrain - MERIT (YAMAZAKI et al. 2017). Visou-se, assim, superar as principais limitações do MGG EGM96 (LEMOINE et al. 1998) e do MDA SRTM (USGS, 2019) originalmente empregados nas reduções e interpolações, bem como distintos SGRs envolvidos inicialmente e não compatibilizados (WGS84 e SGRS67). Também se realizaram depurações de outliers e de redundâncias espúrias das observações originais, gerando as funcionais em grades adequadas. As interpolações de gravidade para os pontos de interesse devem ter por base anomalias da gravidade de Bouguer de acordo com os critérios mais usados no contexto da IAG (FORSBERG; TSCHERNING, 2008). Assim, a partir de anomalias da gravidade interpoladas, foram obtidos os valores da gravidade nos pontos da grade requerida à solução do PVCG. Neste trabalho são discutidos os distúrbios da gravidade em grade. As melhorias com as estratégias adotadas foram significativas. Todos os detalhes dos cálculos, testes de consistência dos dados reduzidos e análises encontram-se na sequência deste artigo.

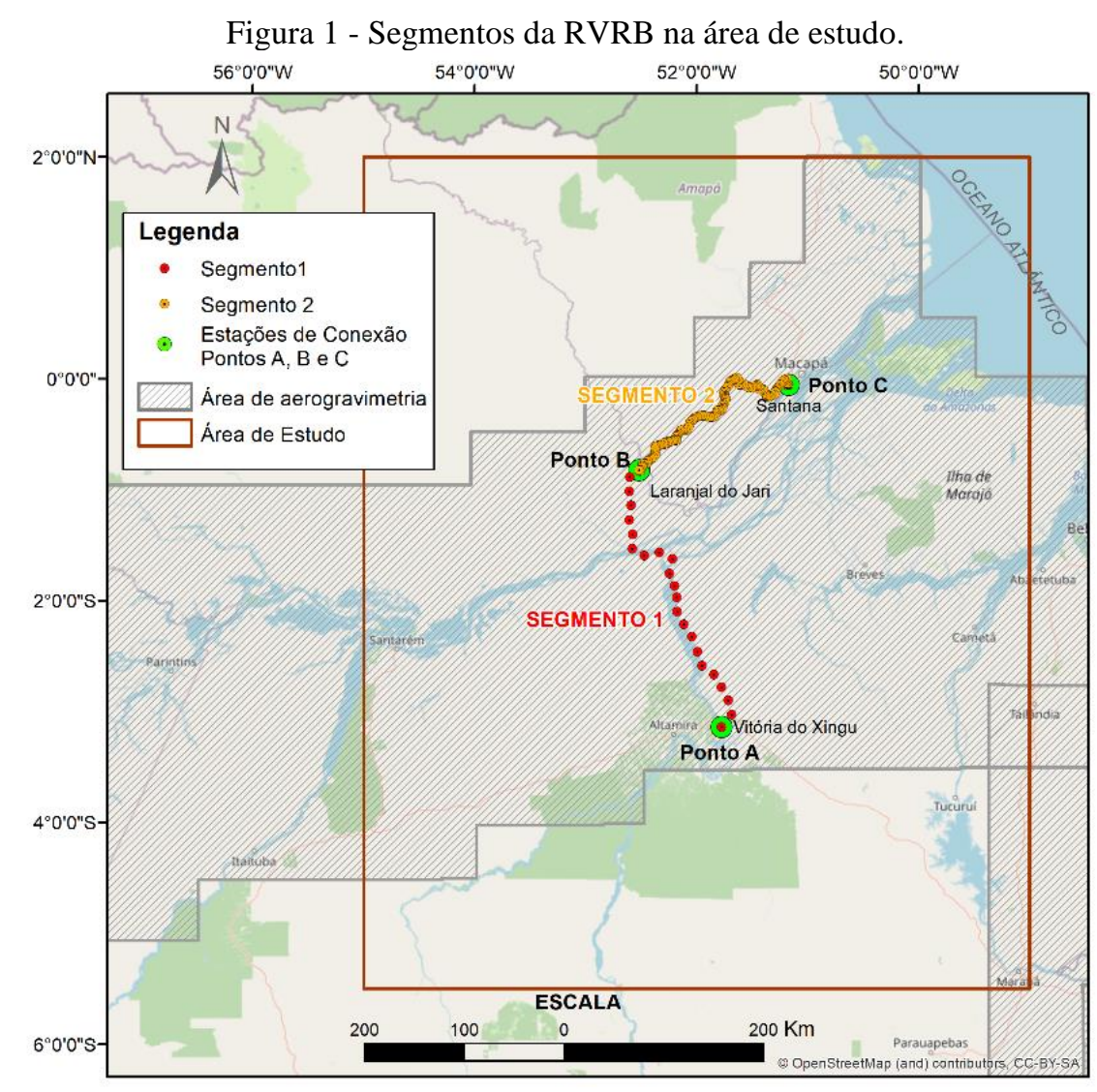




\section{Fonte: Os autores (2021). \\ 2 GERAÇÃO DE BASE DE DADOS A PARTIR DA AEROGRAVIMETRIA}

\subsection{Principais características da base de dados aerogravimétricos originais}

Para a solução do PVCG na sua forma livre e fixada, é relevante o uso de todos os possíveis dados de gravidade e GNSS existentes na zona de estudo. Destaque-se que com a colaboração do Banco Nacional de Dados Gravimétricos - BNDG, da ANP e com o auxílio do IBGE, foi possível ter acesso ao "Aerolevantamento Gravimétrico \& Magnetométrico", projeto desenvolvido em março de 2006, para a identificação de áreas com ocorrência potencial de petróleo e gás nas bacias do Amazonas, Marajó e adjacências. Este levantamento tem uma grande quantidade de dados aerogravimétricos com aplicação na Geofísica, e por se concentrar na área de estudo da presente pesquisa, torna-se assim a principal base de dados utilizada.

É importante conhecer algumas das especificidades do levantamento (ANP, 2008):

a) foram utilizados 3 sensores gravimétricos aeroembarcados modelo GMB3. A aquisição do dado aconteceu com um espaçamento de aproximadamente 18,7 m entre pontos de medição, em função de uma taxa de amostragem de $24 \mathrm{~Hz}$ por sensor, tendo uma precisão declarada melhor do que 0,8 mGal para filtragem de 120s. A referência do levantamento relaciona-se com estações da Rede Gravimétrica do Brasil vinculadas à IGSN1971;

b) foi utilizado o método GPS diferencial dupla frequência com precisão declarada melhor de 1 $\mathrm{cm}$ no sentido horizontal e com precisão melhor de $10 \mathrm{~cm}$ no posicionamento altimétrico;

c) os Sistemas de Referência do levantamento foram o SAD69 e WGS84;

d) as altitudes de amostragem das observações foram aproximadamente a $1400 \mathrm{~m}$ acima do elipsoide WGS84;

e) a distribuição dos dados aerogravimétricos segue as linhas de voo estabelecidas para o projeto, com o qual foram levantados mais de oito milhões e seiscentos mil pontos;

f) o espaçamento entre Linhas de Medição (sentido Leste-Oeste) foi de aproximadamente 6000 $\mathrm{m}$, enquanto o espaçamento de Linhas de Controle (sentido Norte-Sul) foi de $\sim 24000 \mathrm{~m}$.

Uma análise completa dos dados aerogravimétricos e seu uso potencial para fins geodésicos pode ser encontrada em Jesus e De Freitas (2020). Aqui foram analisados e comparados valores derivados da gravidade observada na altura de voo contra valores obtidos mediante MGG EGM96 e MDA SRTM, o qual teve como conclusão de que as funcionais anomalias da gravidade Free-Ar e de Bouguer, advindas do levantamento aerogravimétrico original, não podem ser aplicadas para fins geodésicos, por apresentar inconsistências tanto no uso dos elipsoides de referência, nas superfícies de redução, no cálculo de algumas funcionais e na desconsideração do Sistema de Maré Permanente - SMP necessários às reduções altimétricas (IHDE et al. 2017). Para o aproveitamento do levantamento, foram determinados valores da gravidade reduzidos à SF adequada em função dos valores de gravidade na aeronave (dados observados "brutos" nos sensores embarcados). Foram realizadas todas as compatibilizações de SGRs, SMPs e reduções à SF, dentro dos preceitos mais atuais da Geodesia, conforme apresentado na sequência deste trabalho. Desta forma, visou-se determinar funcionais do campo da gravidade como as características desejáveis para finalidades geodésicas.

Em paralelo com o estudo apresentado anteriormente, foi realizado outra análise e depuração específica dos dados, devido que foram encontradas algumas incongruências em algumas linhas de voo como: segmentação da linha sem sequência nos valores ou de gravidade ou de altitude de voo; valores muito diferentes entre pontos de quase as mesmas coordenadas, entre outros. Os erros referidos foram evidenciados ao gerar uma superfície em função dos valores de gravidade e altitude da aeronave. Toda essa depuração é apresentada na Sessão 2.2.3. 


\subsection{Estratégias para a geração da base de dados para solução do PVCG}

\subsubsection{CARACTERIZAÇÃO DA REGIÃO DE CONEXÃO E DELIMITAÇÃO DA BASE DE DADOS}

Inicialmente foram definidos os pontos A, B e C, como Estações de Conexão - ECs, as quais determinam o início e o fim dos segmentos a conectar dentro da RVRB (Ver Figura 2). Estas estações contêm dados de nivelamento referidos a diferentes segmentos da RVRB, dados de posicionamento GPS em GRS80 e gravimetria em IGSN71, que servirão para avaliação do offset entre os segmentos em função das funcionais objetos de discussão no presente trabalho. Para o estabelecimento de grades de funcionais a serem utilizadas na solução PVCG, foram definidos raios de integração de: $0,5^{\circ} \sim 55 \mathrm{~km}, 1^{\circ} \sim 110 \mathrm{~km} \mathrm{e} 2^{\circ} \sim 220 \mathrm{~km}$ ao redor das ECs segundo a metodologia para a determinação de estações "IHRF" apresentada por Sánchez (2016).

Figura 2 - Definição da área de estudo em função da distribuição do levantamento aerogravimétrico e de raios de integração.

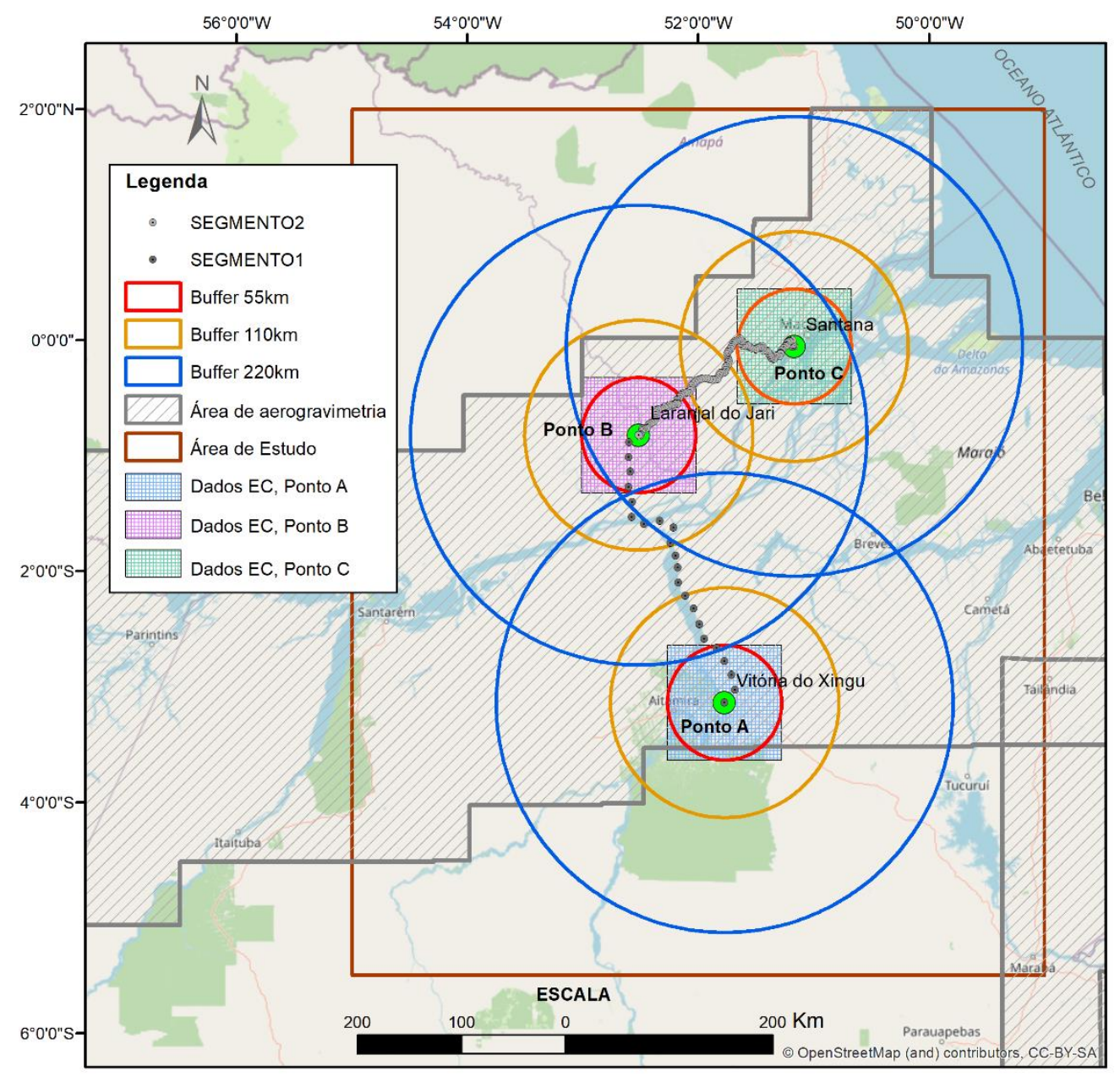

Fonte: Os autores (2021).

Devido à falta de dados ou à distribuição heterogênea destes, o uso de cada raio de integração poderia variar em cada EC. Entretanto, para uma correta análise em função da distribuição dos dados (Ver Figura 2), julgou-se que a melhor cobertura que abrange as três ECs, é o raio de integração de $0,5^{\circ}$. Também é importante considerar que a área de estudo se encontra numa região onde foram detectados gradientes anômalos que certamente influenciam as funcionais envolvidas e têm alcance nos resultados dos cálculos (SANTACRUZJARAMILLO; DE FREITAS; LUZ, 2019). Este aspecto é marcante no segmento 1 (ECs A-B).

Para cada EC realizou-se uma análise individual:

a) a EC-A vinculado ao do DV-I, a qual contém a maior porção da RVRB. Na sua região, não 
existe uma boa distribuição de dados gravimétricos, porém os dados aerogravimétricos são os únicos disponíveis com razoável distribuição regional. Portanto, o uso de um raio de integração maior do que $0,5^{\circ}$, poderia gerar uma distorção na interpolação e geração de grades e por consequência, nos cálculos;

b) a EC-B é um ponto de cálculo fundamental para determinar o offset existente entre os dois segmentos da RVRB. A distribuição e quantidade de dados gravimétricos na sua região é satisfatória já que a grade de $1^{\circ} \times 1^{\circ}$ em função do raio de integração adotado de $0,5^{\circ}$, é $100 \%$ atendida na cobertura dos dados aerogravimétricos;

c) a EC-C é a estação mais próxima à DV-S, a qual vincula-se ao segmento da RVRB pertencente a Santana. Tem uma boa distribuição e preenchimento dos dados no raio de integração $0,5^{\circ}$, entretanto se encontra numa área com maior influência de água (doce e salgada) e no limite costeiro, fatores que implicam de forma geral em perturbações nos dados oriundos dos MGGs e MDAs.

\subsubsection{COMPATIBILIZAÇÃO DE SISTEMAS GEODÉSICOS DE REFERÊNCIA E SISTEMAS DE MARÉS PERMANENTES}

O levantamento aerogravimétrico (ANP), tem um papel fundamental na presente pesquisa. Graças à densidade e a distribuição de dados disponíveis, pode-se elaborar grades homogêneas com valores de distúrbios da gravidade e de anomalias de Molondensky (muitas vezes referidas como anomalias ar livre de superfície do inglês free-ar surface anomalies) a serem aplicados na solução do PVCG. Para isto, é imprescindível a redução dos valores da gravidade levantada na aeronave para a SF da Terra.

Para trabalhar inicialmente com a aerogravimetria, foi necessária uma depuração prévia da base de dados fornecida para adequar-se as observações aos SGRs, SMPs previsto nos preceitos atuais na Geodesia (IAG, 2015a,b). Originalmente, as observações posicionais foram vinculadas ao WGS84 (e alternativamente ao SAD69). Os aspectos vinculados ao geopotencial por exemplo, a gravidade normal, foram referidos no levantamento original ao GRS67 e não foram discutidas as correções em função do SMP e superfícies de redução utilizadas não são aquelas adequadas para fins geodésicos.

Para realizar a redução da gravidade à SF, foi necessário analisar as diferentes superfícies de referência, fixar o SGR GRS80 como base de todas as referências posicionais e do geopotencial, e as funcionais agora determinadas. Estes aspectos são discutidos na sequência deste artigo, que têm por base preceitos e modelos fornecidos por técnicas espaciais modernas. Uma visão geral dos aspectos trabalhados e as reduções efetivadas para determinar a gravidade sobre a SF e desta as grades mencionadas, é apresentada na Figura 3.

Figura 3 - Funcionais para a determinação da gravidade na SF.

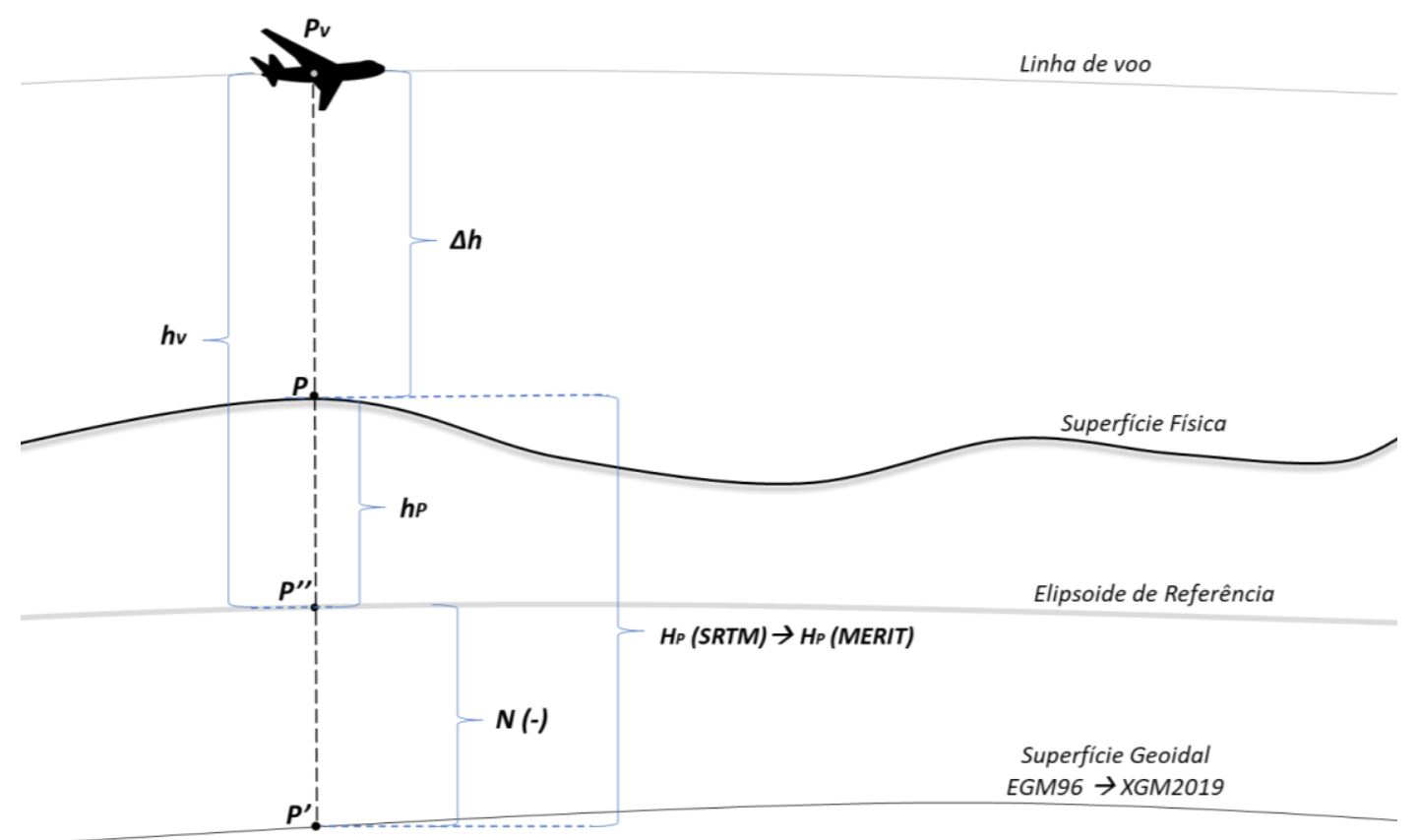

Fonte: Os autores (2021). 
Foram efetivados grupos de cálculo para cada EC (A, B e C), e definido o raio de integração a ser usado, já que não seria possível trabalhar com tão extenso volume de dados aerogravimétricos (Figura 2). Inicialmente foi considerado o SRTM3 com resolução espacial de 3" de arco, aproximadamente 90 metros (derivado do SRTM1) para a determinação da altitude ortométrica $H_{P(S R T M)}$. Como as funcionais encontramse em diferentes superfícies de referência, foi necessária a determinação dos valores de altitude geoidal $N$, com respeito ao modelo EGM96, já que este é o SGR vertical do SRTM3. Com os valores de $H_{P_{S R T M}}$ e $N_{E G M 96}$, determinou-se a altitude elipsoidal $h_{P}$ em cada ponto sobre o elipsoide de referência (para abreviar as equações, considerar o ponto $\mathrm{P}$ como representativo de todos os pontos envolvidos). Para este caso, foi considerado o WGS84 (com SMP em Tide Free - TF), já que este elipsoide foi considerado na aerogravimetria e é o SGR horizontal do SRTM3. Assim:

$$
h_{P}=H_{P_{S R T M}}+N_{E G M 96}
$$

Segundo os preceitos do IHRS/IHRF (IAG, 2015a) deve-se trabalhar com o elipsoide de referência do GRS80 e seus demais parâmetros, no SMP Mean Tide - MT para as posições verticais e Zero Tide - ZT para as grandezas associadas ao geopotencial. É importante destacar que todos os produtos gerados serão transformados para o Sistema MT, possibilitando assim, análises em um único SMP. Pode-se observar na Figura 3, que as funcionais se encontram em diferentes referenciais. Portanto, devem ser compatibilizados a um único SGR e SMP. Para isto, foi modelada uma superfície de transformação envolvendo todas as relações de altitude considerados os SGRs, SMP e MGGs envolvidos. Assim, considera-se a altitude geoidal $N_{M G G_{\text {novo }}}$ e altitude ortométrica $H_{M G G_{\text {novo }}}$ proveniente de um novo MGG, a $N_{E G M 96}$ e $H_{E G M 96}$ são derivados do EGM96 e, a diferença de altitudes elipsoidais referidas aos elipsoides de referência envolvidos. Desta forma, considerando-se para os pontos amostrados:

$$
\left(N_{M G G_{\text {novo }}}-N_{E G M 96}\right)=\left(h_{G R S 80}-h_{W G S 84}\right)-\left(H_{M G G_{\text {novo }}}-H_{E G M 96}\right)
$$

tem-se a possibilidade de gerar uma superfície que modela a transformação necessária na altitude para qualquer ponto dentro da região considerada.

Para a transformação entre elipsoides de referência foi usada a fórmula empírica (RENGANATHAN, 2010):

$$
\left(h_{S G R 2}-h_{S G R 1}\right)=\delta h=-\left(\left(a_{S G R 2}-a_{S G R 1}\right) * \cos (\varphi)^{2}+\left(b_{S G R 2}-b_{S G R 1}\right) * \operatorname{sen}(\varphi)^{2}\right)
$$

onde $a$ e $b$ representam o semieixo maior e semieixo menor dos dois elipsoides de referência da transformação, respectivamente.

Da superfície de transformação, foi utilizada a $H_{M G G_{\text {novo }}}$ da Eq. (2) para o novo cálculo de $h_{P}$, que já está compatibilizado conforme aos preceitos desejados. Assim, todos os cálculos subsequentes baseiam-se em:

$$
h_{P_{M G G \text { novo }}}=H_{P_{M G G \text { novo }}}+N_{P_{M G G} \text { novo }}
$$

Note-se que o $M G G_{\text {novo }}$ pode ser substituído por diferentes MGGs, como é apresentado na Sessão 2.2.3.

Conforme a Figura 3, os dados aerogravimétricos forneceram a altitude de voo $h_{v}$ de cada ponto levantado em WGS84. Com isto, determinou-se a $\Delta h$, que é o valor de altitude com base na $h_{P_{M G G} \text { novo }}$, a qual é necessária para o cálculo da gravidade no ponto $\mathrm{P}$ na SF.

$$
\Delta h=h_{P_{M G G \text { novo }}}-h_{v}
$$


Com o valor de $\Delta h$ pode-se realizar a redução da gravidade ao ponto $\mathrm{P}$, aplicando o gradiente vertical da gravidade normal, obtendo $g_{P}$ com os valores da aerogravimetria $g_{v}$ :

$$
g_{P}=g_{v}+\frac{\partial \gamma}{\partial h} \Delta h
$$

onde o gradiente vertical da gravidade normal é dado por (HEISKANEN; MORITZ, 1985, pp. 86):

$$
\frac{\partial \gamma}{\partial h}=\frac{2 \gamma}{a}\left(1+f+m-2 \operatorname{sen}^{2} \varphi\right)
$$

Com os valores da gravidade reduzidos à SF em cada ponto da aerogravimetria, é possível o cálculo de anomalias de Bouguer $\left(\Delta g_{B}\right)$ conforme mostra a Eq. (8) (HOFMANN-WELLENHOF; MORITZ, 2006, pp. 136). As anomalias de Bouguer são consideradas como as funcionais mais apropriadas para interpolações em vista da suavidade de sua variabilidade espacial. Assim, são base para interpolação da gravidade na SF em uma grade regular, e a partir destes valores podem ser gerados outras funcionais adequadas para a solução do PVCG, nas Estações de Conexão.

$$
\Delta g_{B}=g_{P}+0,3086 H_{P_{M G G \text { novo }}}-0,1119 H_{P_{M G G \text { novo }}}-\gamma_{P^{\prime \prime}}
$$

onde $\gamma_{P^{n}}$ representa a gravidade normal que pode ser calculada pela fórmula Somigliana ou pelo teorema de Clairaut (ver mais em HOFMANN-WELLENHOF; MORITZ, 2006, p. 71-72 e 86).

\subsubsection{TESTES DE ADERÊNCIA DA SUPERFÍCIE DE TRANSFORMAÇÃO COM BASE EM MGGs E MDAs}

Inicialmente realizou-se a plotagem da superfície gerada a partir das altitudes das estações do aerolevantamento. Com isto foi possível realizar uma análise visual de perturbações na superfície, possibilitando a detecção de possíveis outliers. Mediante a geração de superfícies dos valores da altitude de voo $\left(h_{v}\right)$, foram constatados alguns erros especificamente nas regiões de cálculo das estações de conexão A e $\mathrm{C}$ (a Figura 4 mostra a EC-A). Como a $h_{v}$ tem relação inversa com a gravidade na aeronave, eliminou-se estes outliers da base de dados. Estas incongruências podem ser atribuídas a vários fatores como: mudanças das linhas de voo no levantamento aerogravimétrico; sobreposição de linhas de voo com diferentes valores de altitude e gravidade devido à transição do levantamento; regiões com perturbações nas observações baseadas em DGPS; entre outros. Na Figura 4 pode-se notar o comportamento da topografia na região da EC-A, sendo que especificamente a Figura 4(b), representa a topografia determinada pelo MERIT. Com o qual podemos observar que as grandes perturbações das linhas de voo apresentadas na Figura 4(a) não justificam os saltos observados, já que estes não acompanham o relevo. 
Figura 4 - Depuração dos dados na região da EC-A. (a) altitudes que variam de 1293,2 m a 1540,1 m: (b) altitudes no terreno variando de 0,2 m a 252,8 m; (c) altitudes de voo após depuração com variação de $1381 \mathrm{~m}$ a $1437,5 \mathrm{~m}$.

(a) Altitude de voo antes da depuração na região da EC-A

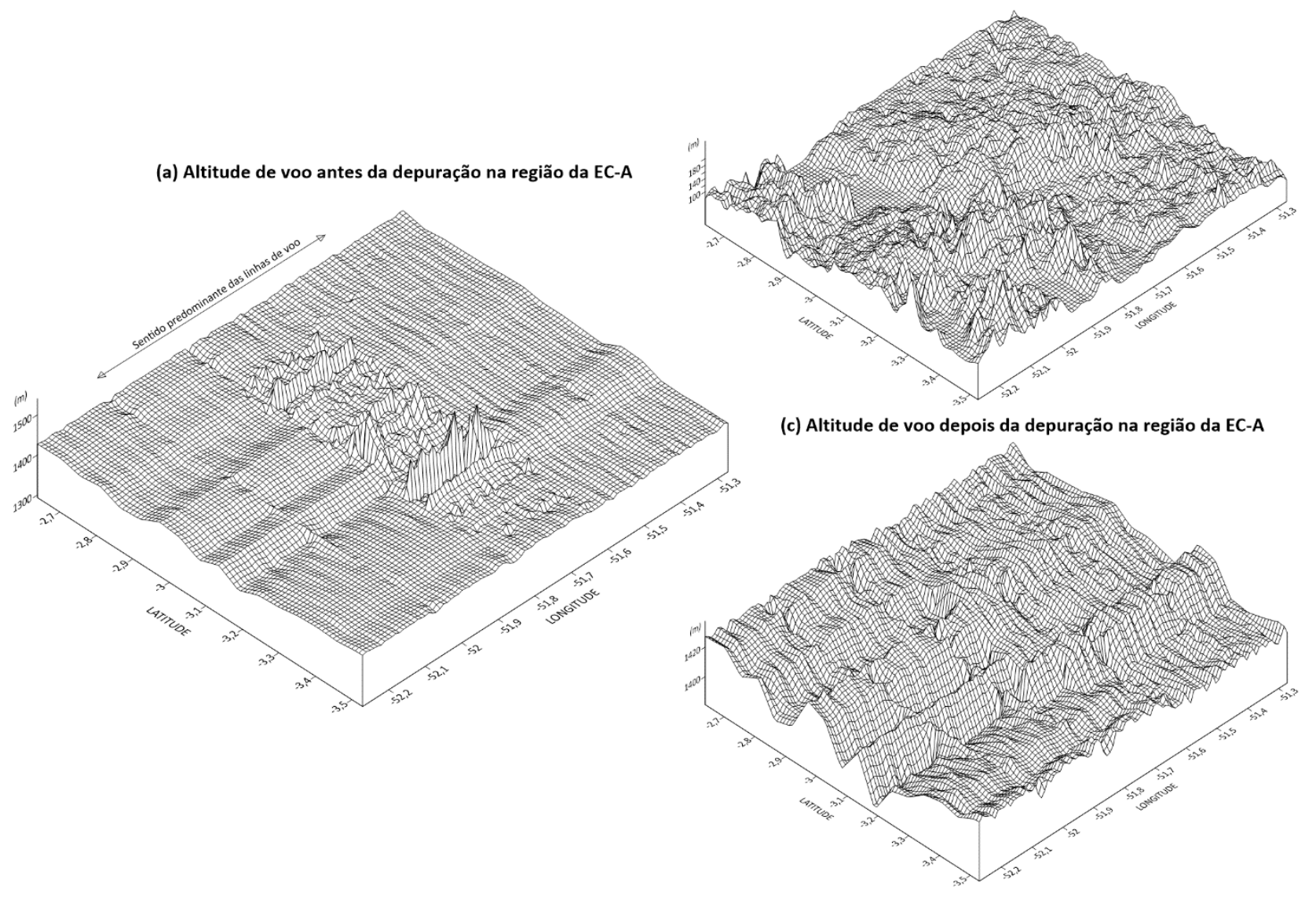

(b) MDA MERIT na região da EC-A

Fonte: Os autores (2021).

A depuração foi realizada manualmente, eliminando as faixas com mais perturbações e incoerências nos valores dos dados. Os outliers detectados:

a) a região de cálculo da EC-A $\left(1^{\circ} \times 1^{\circ}\right)$ inicialmente tinha 167.954 pontos antes da depuração, sendo que depois foram eliminados 25.417 pontos associados com as anomalias mostradas na Figura 4. Portanto, foram utilizados 142.537 pontos para a determinação de anomalias de Bouguer;

b) na região de cálculo da EC-B não foram encontrados outliers expressivos. Os cálculos, portanto, foram realizados com 169.022 pontos;

c) originalmente a região de cálculo da EC-C continha 190.299 pontos de cálculo, depois da depuração, ficaram 168.084 pontos.

As funcionais originais geradas no levantamento para a ANP possuíam erros sistemáticos advindos principalmente de reduções nas altitudes e a não consideração dos efeitos das marés permanentes. Como teste inicial das reduções gravimétricas no presente estudo, recorreu-se à comparação das $\Delta g_{B}$ calculadas com os novos valores da gravidade reduzida à SF $\left(g_{P}\right)$ e das anomalias da gravidade correspondentes oriundas do MGG EGM2008 (PAVLIS. et al. 2008). Foram realizados três testes baseados na geração de superfícies de transformações estabelecidas em função dos SGRs envolvidos e MGGs conforme apresentado na sequência:

a) o teste 1 considera uma superfície de transformação com o SGR (WGS84) e SMP (TF) dos dados aerogravimétricos originais, portanto, sua única variante é a transformação entre MGGs (EGM96 $\rightarrow$ EGM2008). Este teste serve de base para avaliar os testes 2 e 3, demostrando assim a existência de importantes variações quando alterados os SGRs e SMP para adequação ao Sistema Geodésico Brasileiro (SGB);

b) o teste 2 considera transformação de SGRs (WGS84 $\rightarrow$ GRS80), no mesmo SMP (TF) usando 
o EGM2008 para a transformação EGM96 $\rightarrow$ EGM2008;

c) o teste 3 utiliza uma superfície de transformação modelada com base na conversão entre SGRs (WGS84 $\rightarrow$ GRS80) e SMP (TF $\rightarrow$ MT), e usando o EGM2008 para a transformação EGM96 $\rightarrow$ EGM2008.

A maior variação na transformação (Eq. 2) decorre de $\Delta N=N_{M G G_{n o v o}}-N_{E G M 96}$, já que para o cálculo deste funcional necessita-se de informação tanto do MGG, do SGR como do SMP. O $\delta h$ entre os SGRs WGS84 e GRS80, é obtido com a Eq. (3). Desta forma, para desenvolver os testes foi indispensável a análise dos $\Delta N_{S}$ de cada teste, considerando suas especificidades. Na Figura 5 apresenta-se em forma mais detalhada os diferentes $\Delta N_{S}$ calculados, cujos resultados estão apresentados na Tabela 1.

Figura 5 - Determinação de $\Delta N$ em função das especificidades dos testes.

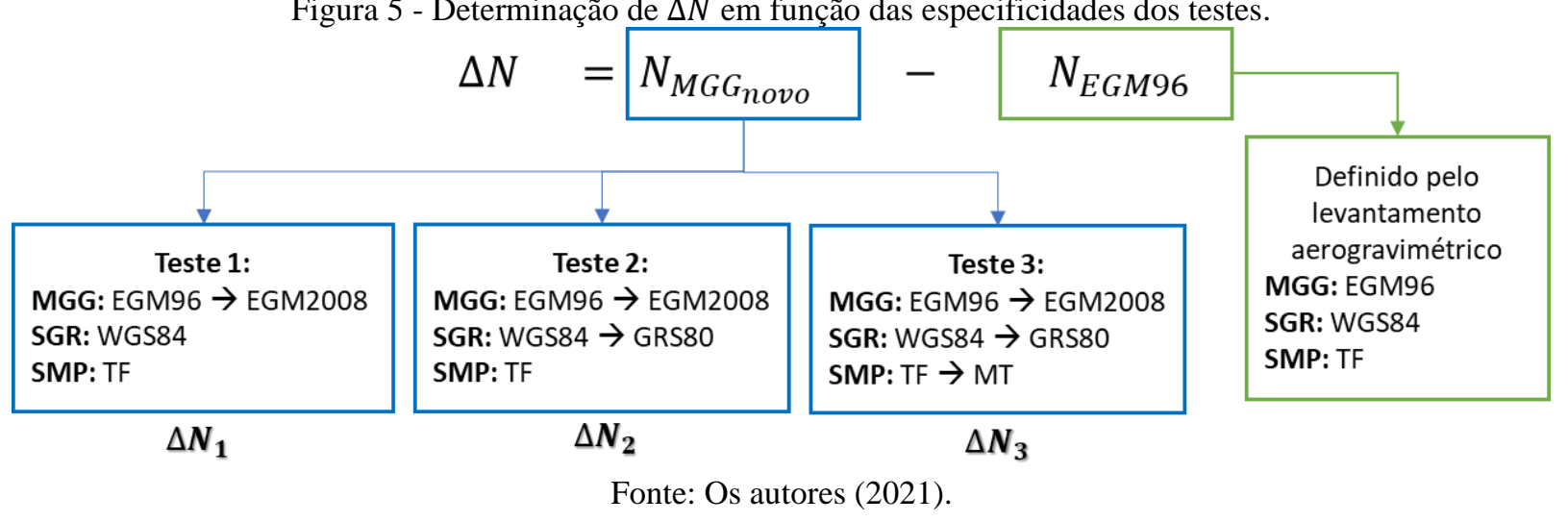

Nos testes iniciais, foi considerado o MDA SRTM3 para a obtenção do $H_{P_{S R T M}}$, assim como também o EGM2008 como o novo MGG, devido ser este um modelo combinado bastante empregado no Brasil (MELO; DE FREITAS, 2012; NICACIO; DALAZOANA; DE FREITAS, 2018), inclusive foi a base para o desenvolvimento do modelo de ondulação geoidal oficial do Brasil, o MAPGEO2015 (DE MATOS et. al, 2016). Além do descrito, o EGM2008 tem uma resolução espacial de aproximadamente $9 \mathrm{~km}$, correspondente ou grau de desenvolvimento 2190 e ordem 2159 em harmônicos esféricos. Uma avaliação completa deste MGG, incluindo a região de estudos, é realizada por Pavlis. et al. (2012).

Para avaliar estes primeiros testes, foram calculadas as médias para os $\Delta N_{s}$ correspondentes a cada $M G G_{N O V O}$ de acordo com o exposto na Figura 5. As estatísticas para o $\Delta N_{1}$ (teste 1), $\Delta N_{2}$ (teste 2) e $\Delta N_{3}$ (teste 3) são apresentadas na Tabela 1. Pode-se observar que as médias do teste 2 e teste 3 não têm muita variação, porém a conversão já aporta uma correção de aproximadamente $2 \mathrm{~m}$ nas altitudes, mesmo sem considerar a contribuição do resto das funcionais envolvidas na Eq. (2). Foi considerado o teste 3 como o teste "ideal" já que este apresenta a menor média, além de conter no cálculo o SGRs GRS80 e SMP MT, e com isto tem-se a base para testes com outros MGGs mais atuais, respeitando as características do SGB.

Tabela 1 - Diferenças dos $\Delta N_{1}$ na região da EC-A.

\begin{tabular}{c|c|c|c}
\hline Avaliação & Teste 1, $\Delta \boldsymbol{N}_{\mathbf{1}}(\mathbf{m})$ & Teste 2, $\Delta \boldsymbol{N}_{\mathbf{2}}(\mathbf{m})$ & Teste 3, $\Delta \boldsymbol{N}_{\mathbf{3}}(\mathbf{m})$ \\
\hline Mínimo & $-2,546$ & $-3,481$ & $-3,353$ \\
Máximo & $-0,528$ & $-1,462$ & $-1,334$ \\
Média & $-1,301$ & $-2,235$ & $-2,107$
\end{tabular}

Fonte: Os autores (2021).

Em vista de estudos recentes (HIRT; YANG; KUHN, 2019) buscou-se testar outro MDA: o MERIT (YAMAZAKI et al. 2017). Este MDA foi desenvolvido removendo várias fontes de erros (absolute bias desvios absolutos; stripe noise - ruídos de devidos à geometria de aquisição; speckle noise - ruídos pontuais; tree height bias - variações devidas às copas das árvores) presentes no SRTM3 v2.1, com o objetivo de melhorar as altitudes modeladas. O MDA MERIT é, portanto, bastante promissor para a região dos estudos em vista de corrigir efeitos da cobertura florestal, assim como apresentar melhor resposta relativa a corpos d'água com densidade bastante elevada na região, os quais não são adequadamente modelados pelo SRTM. 
Por tratar-se de uma região com topografia predominantemente plana, o SRTM apresenta declives não explicados (YAMAZAKI et al. 2017; HAWKER et al. 2018). Por exemplo, pode-se constatar uma média de aproximadamente $10 \mathrm{~m}$ das diferenças entre $H_{P_{M E R I T}}$ e $H_{P_{S R T M}}$ no entorno da EC-A. O MERIT apresenta algumas especificações iguais ao SRTM3, como sua resolução espacial e os SGRs de base. Deste modo, podese usar as mesmas reduções feitas com base no teste 3 .

Na sequência, foram realizados três testes adicionais. Foram utilizados dois MGGs combinados mais atuais além do EGM2008: o EINGEN-6C4 (FÖRSTE, et al. 2014); e o XGM2019 (ZINGERLE et al. 2019), ambos com grau de desenvolvimento 2190. Estes testes foram realizados com base no $\Delta N_{3}$ do teste 3 já referido, porém com o uso do MERIT. Desta forma, foram calculadas as $\Delta g_{B}$ conforme a Eq. (8) para cada novo MGG. Na Tabela 2 são apresentados os RMS que foram calculados das diferenças entre as $\Delta g_{B_{\text {calculadas }}}$ e as $\Delta g_{B_{M G G}}$ :

Tabela 2 - RMS dos testes para a determinação da superfície de transformação com diferentes MGGs para a EC-A.

\begin{tabular}{c|c}
\hline Testes - MGGs & $\mathbf{R M S}\left(\Delta \boldsymbol{g}_{\left.\boldsymbol{B}_{\text {MGGs }}-\Delta \boldsymbol{g}_{\boldsymbol{B}_{\text {cal }}}\right)}\right)$ \\
\hline (mGal)
\end{tabular}

Fonte: Os autores (2021).

Como pode ser observado na Tabela 2, o teste 6 (que utiliza o MGG XGM2019 nos cálculos) é o que tem o menor RMS, e, portanto, entende-se ser ele o mais adequado para ser utilizado na modelagem da superfície de transformação. Deve ser destacado que o RMS contém erros inerentes ao levantamento e também aqueles da resolução espectral do MGG, menor que a do levantamento aerogravimétrico. De todos os testes efetivados, são aqui apresentados os realizados para a EC-A, já que em sua região concentram-se os maiores problemas detectados nos levantamentos aerogravimétricos. As análises para a EC-A permitiram estabelecer a melhor estratégia de modelagem da superfície de transformação, que é um dos aspectos centrais deste trabalho. Esta estratégia é certamente a mais adequada não só para aplicação nas EC-B e EC-C, assim como também em toda área do levantamento aerogravimétrico.

\subsubsection{GERAÇÃO DE GRADE DE DISTÚRBIOS DA GRAVIDADE A PARTIR DE ANOMALIAS DE BOUGUER}

Uma vez calculadas as $\Delta g_{B}$ nos conjuntos de dados de cada ECs, foi utilizado o programa GEOGRID do pacote computacional GRAVSOFT (FORSBERG; TSCHERNING, 2008) para a geração de uma grade regular de anomalias de Bouguer, com um espaçamento de 1 'x1' dentro de uma área de $1^{\circ} \mathrm{x} 1^{\circ}$ (ver Figura 2). A partir desta grade interpolada, obtém-se os valores da gravidade para os respectivos pontos na superfície. Com a gravidade, assim interpolada, obtém-se as grades de distúrbios da gravidade $\left(\delta g_{P}\right)$ e anomalias de Molodensky $\left(\Delta g_{B}\right)$, sem o uso de hipóteses simplificativas.

Foram realizados alguns testes para identificar os melhores parâmetros para o uso do GEOGRID, lembrando que estes parâmetros devem variar de acordo com o tipo de funcional a ser interpolado, do método utilizado para a interpolação, da distância para selecionar os pontos de cálculo, da quantidade e distribuição de dados de entrada utilizados, entre outros. Na presente pesquisa foi utilizado o método Colocação por Mínimos Quadrados associado com Kriging (FORSBERG; TSCHERNING, 2008), já que se pode calcular a funcional em função de parâmetros determinísticos e estocásticos (sinal e ruído) inseridos no cálculo.

A Tabela 3 apresenta alguns exemplos na diferença dos resultados em função da variação dos parâmetros de distância de correlação/ruído (DC/R), usados no método de cálculo. Note-se que os RMS foram calculados das diferenças entre as $\Delta g_{B_{\text {calculadas }}}$ e as $\Delta g_{B_{M G G S}}$ da grade interpolada no GEOGRID, como apresenta as Figuras 6(a), 6(b) e 6(c). Como pode-se observar, existem diferenças no RMS para cada MGG utilizado como base de análise. Como foi descrito na Sessão anterior, trabalhou-se com as $\Delta g_{B}$ calculadas pelo XGM2019, e para o caso das $\Delta g_{B}$ interpoladas, continuou-se trabalhando com o teste 6 , considerando distâncias de correlação de 15 e $25 \mathrm{~km}$ as quais aparentam envolver uma quantidade mais adequada e melhor 
distribuída de dados de base para interpolação, em vista das distâncias entre linhas voo e disponibilidade de dados na região. A seleção do nível de ruído $5 \mathrm{mGal}$ e $10 \mathrm{mGal}$ foi adotada em vista dos resultados obtidos para o teste 6. Observe-se na Tabela 3 que os melhores resultados obtidos foram para o XGM2019 respectivamente com distância de correlação de $25 \mathrm{~km}$ e nível de ruído de $10 \mathrm{mGal}$.

Tabela 3 - Variação do RMS em função dos parâmetros utilizados no GEOGRID em Grade interpolada para anomalias de Bouguer.

\begin{tabular}{|c|c|c|}
\hline \multirow[t]{2}{*}{ MGGs } & \multicolumn{2}{|c|}{$\begin{array}{c}\text { RMS da Grade interpolada } \\
\text { (mGal) }\end{array}$} \\
\hline & DC/R (15.0/5.0) & DC/R (25.0/10.0) \\
\hline EGM2008 & 11,29 & 11,38 \\
\hline EINGEN-6C4 & 10,49 & 10,13 \\
\hline XGM2019 & 8,04 & 6,85 \\
\hline
\end{tabular}

Fonte: Os autores (2021).

Na sequência do cálculo das $\Delta g_{B}$, foram recuperados os valores da gravidade reduzida em cada ponto da grade segundo a Eq. (8), e determinados os distúrbios da gravidade $\left(\delta g_{P}\right)$ de acordo com a Eq. (9):

$$
\delta g_{P}=g_{P}-\gamma_{P}
$$

onde a $\gamma_{P}$ é a gravidade normal no ponto P. Destaque-se que esta grandeza é fundamental para a solução do PVCG, pois possibilita uma condição de contorno, a qual estabelece uma relação direta entre o potencial perturbador e o distúrbio da gravidade (HOFMANN-WELLENHOF e MORITZ, 2006, p. 115). Uma vez calculados os $\delta g_{P}$ oriundos das observações, pode-se calcular $\delta g_{R E S}$ em função de MGGs, e MDAs, mediante a Técnica Remove-Restore, por exemplo, e assim calcular a solução do PVCG.

Efetivou-se os cálculos dos RMSs dos distúrbios da gravidade e anomalias de Molodensky obtidas a partir das reduções efetivadas com base nos valores modelados pelo XGM2019. O RMS dos distúrbios da gravidade $\left(\delta g_{P}\right)$ foi de $6,65 \mathrm{mGal}$ e o RMS para anomalias de Molodensky $\left(\Delta g_{M}\right)$ foi de 5,38 mGal. Estes valores de RMS são consistentes com o valor de RMS obtido para as anomalias de Bouguer. Uma visualização para os valores de $\delta g_{P}$ é apresentada nas Figuras 6(d), 6(e) e 6(f). 
Figura 6 - Superfícies de Anomalias de Bouguer e de Distúrbios da gravidade determinadas mediante: dados interpolados (a, d); dados oriundos do MGG XGM2019 (b, e); e RMS das diferenças das superfícies (c, f).

(a) Anomalias de Bouguer interpoladas

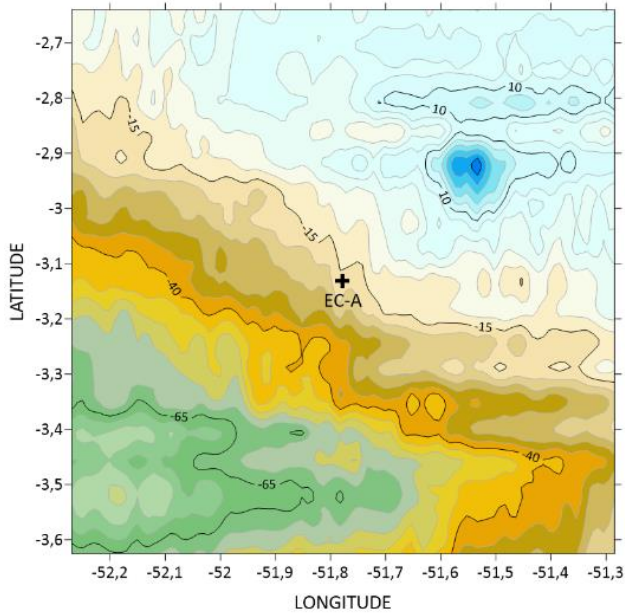

(b) Anomalias de Bouguer do MGG XGM2019

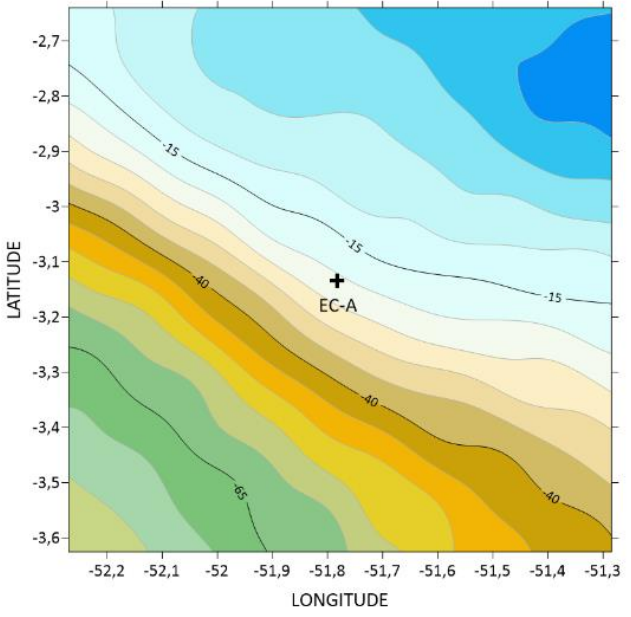

(c) Diferenças de Anomalias de Bouguer (a) - (b)

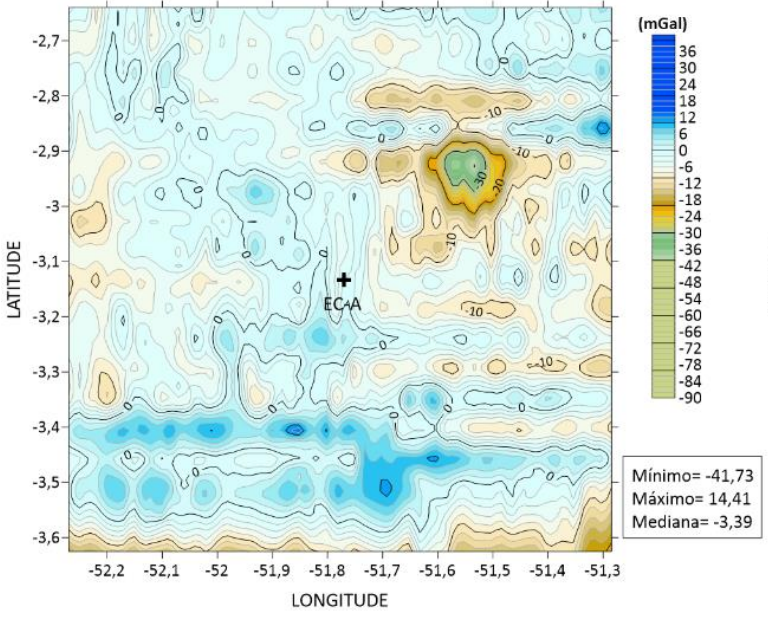

(d) Distúrbios da gravidade interpolados
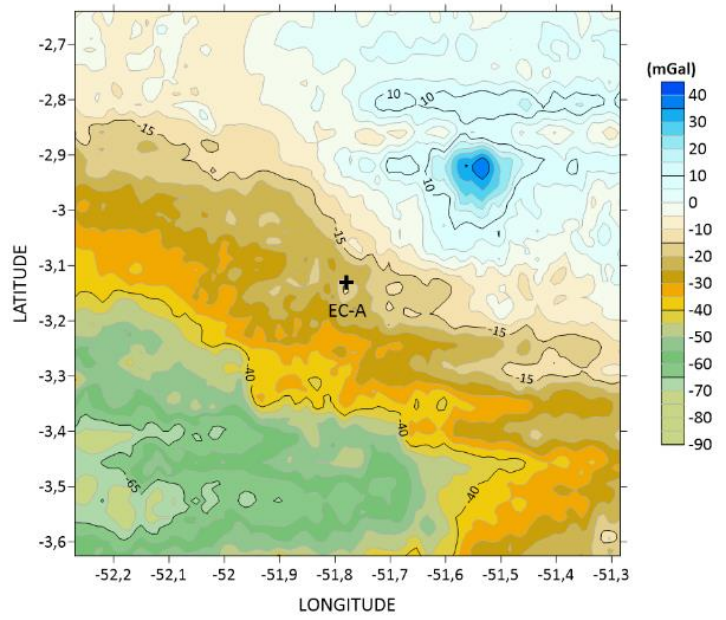

(e) Distúrbios da gravidade do MGG XGM2019

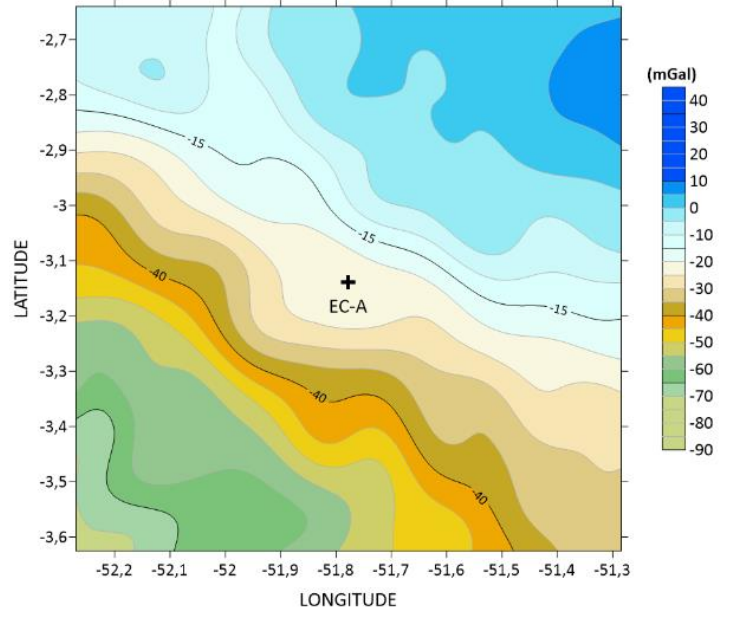

(f) Diferenças de Distúrbios da gravidade (d) - (e)
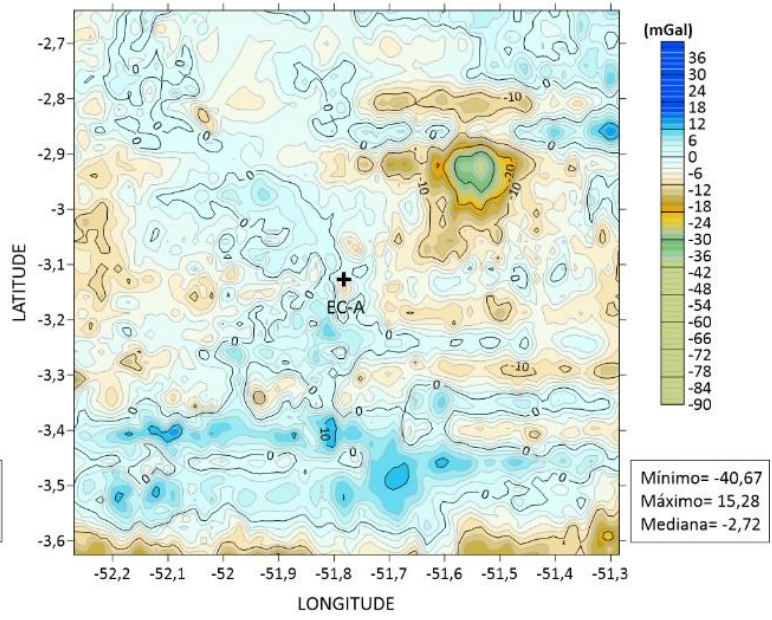

Fonte: Os autores (2021).

\section{DISCUSSÕES E CONCLUSÕES}

Foram buscadas diferentes alternativas para a determinação de funcionais da gravidade a partir da aerogravimetria. Em vista dos referenciais e reduções empregadas nos levantamentos originais para fins geofísicos, foram necessárias diversas estratégias para obtenção de funcionais adequadas para finalidades geodésicas. Foram testadas alternativas de MGGs e MDAs para compatibilização dos SGRs dentro das 
expectativas do SGB, bem como para teste das funcionais obtidas a partir das estratégias adotadas no presente trabalho.

Testou-se os MGGs e MDAs mais atuais da Geodesia. Configuraram-se como mais adequados o MGG XGM2019 e o MDA MERIT.

Efetivou-se as compatibilizações necessárias dos SMPs para todas as funcionais trabalhadas.

Foram realizadas várias etapas para a redução da gravidade observada nos sensores na altitude de voo visando sua redução para a SF, e assim determinar uma grade de anomalias da gravidade de Bouguer e de distúrbios de gravidade, estes a serem empregados na solução do PVCG.

Com a geração da superfície de transformação adequada, obteve-se uma melhora significativa nos resultados, como foi demostrado com o RMS de $6,85 \mathrm{mGal}$ das anomalias da gravidade de Bouguer e 6,65 mGal para os distúrbios da gravidade. Estes valores indicam que a base de dados obtida no contexto deste trabalho, tem uma boa aderência com os pressupostos da Geodesia para emprego em técnicas de decomposição espectral e geração de residuais para emprego na solução do PVCG, de forma independente de SGRs locais.

Todos os testes realizados foram importantes para analisar o comportamento dos dados em função de sua distribuição. Concentrou-se as análises na EC-A, cuja região apresentava as maiores perturbações nos dados adquiridos e geometria menos favorável na distribuição de dados. Entende-se que as estratégias desenvolvidas e aplicadas na EC-A apresentam as melhores condições para gerar grades consistentes nas ECB e EC-C dentro dos preceitos IAG/GGOS/IHRS/IHRF, bem como em toda a área do levantamento.

\section{Agradecimentos}

Os autores agradecem às seguintes instituições: à Secretaria de Educación Superior, Ciencia, Tecnología e Innovación de Ecuador (SENESCYT) pela bolsa concedida à pós-graduanda Andrea Galudht Santacruz Jaramillo; ao Conselho Nacional de Desenvolvimento Científico e Tecnológico do Brasil (CNPq) pelo apoio à Pesquisa; ao Instituto Brasileiro de Geografia e Estatística (IBGE) pelo fornecimento dos dados necessários para o desenvolvimento da pesquisa, em especial à Coordenação de Geodésia e Cartografia e à Universidade Federal do Paraná (UFPR).

\section{Contribuição dos Autores}

A primeira autora desenvolveu a curadoria dos dados com o aval do segundo autor. Ambos os autores participaram durante o resto das etapas da pesquisa como: conceptualização, metodologia, validação, redação, revisão e edição.

\section{Conflitos de Interesse}

Os autores declaram que não há conflitos de interesse.

\section{Referências}

AGÊNCIA NACIONAL DE PETRÓLEOS (ANP). Levantamento Aero-geofísico para a identificação de áreas com ocorrência potencial de petróleo e gás nas bacias do Amazonas, Marajó e Adjacências. Tomo I: Área Amazonas. Aerolevantamento Gravimétrico \& Magnetométrico. São Paulo: ANP, 2008.

BARZAGHI, R.; DE GAETANI, C.I.; BETTI, B. The worldwide physical height datum Project, Rendiconti Lincei. Scienze Fisiche e Naturali, 2020, p. 1-8. DOI. 10.1007/s12210-020-00948-0.

FORSBERG, R.; TSCHERNING, C. C. An overview manual for the GRAVSOFT geodetic gravity field modelling programs. Contract report for JUPEM, 2008.

FÖRSTE, C.; BRUINSMA, S. L.; ABRIKOSOV, O.; LEMOINE, J. M.; MARTY, J. C.; FLECHTNER, F.; BALMINO, G.; BARTHELMES, F.; BIANCALE, R. EIGEN-6C4 The latest combined global gravity field model including GOCE data up to degree and order 2190 of GFZ Potsdam and GRGS Toulouse; GFZ Data Services, 2014. DOI. 10.5880/ICGEM.2015.1. 
HAWKER, L.; ROUGIER, J.; NEAL, J.; BATES, P.; ARCHER, L.; YAMAZAKI, D. Implications of simulating global digital elevation models for flood inundation studies. Water Resources Research, v. 54, n. 10, p. 7910-7928. DOI. 10.1029/2018WR023279.

HEISKANEN, W. A.; MORITZ, H. Physical Geodesy. Springer Wien New York, Madrid, 1985.

HIRT, C.; YANG, M.; KUHN, M.; BUCHA, B.; KURZMANN, A.; PAIL, R. SRTM2gravity: An Ultrahigh Resolution Global Model of Gravimetric Terrain Corrections. Geophysical Research Letters, 2019, v. 46, n. 9, p. 4618-4627. DOI. 10.1029/2019GL082521.

HOFMANN-WELLENHOF, B.; MORITZ, H. Physical Geodesy. Springer Science \& Business Media, 2006.

INTERNATIONAL ASSOCIATION OF GEODESY (IAG). Resolution (No. 1) for the definition and realization of an International Height Reference System (IHRS). München - Germany, 2015a. Disponível em: <https://ihrs.dgfi.tum.de/fileadmin/JWG_2011/IAG_Resolutions_2015.pdf>. Acesso em: fev. 2020.

INTERNATIONAL ASSOCIATION OF GEODESY (IAG). Resolution (No. 2) for the establishment of a Global Absolute Gravity Reference System. München - Germany, 2015b. Disponível em: <https://ihrs.dgfi.tum.de/fileadmin/JWG_2011/IAG_Resolutions_2015.pdf>. Acesso em: fev. 2020.

INTERNATIONAL ASSOCIATION OF GEODESY (IAG). Description of the Global Geodetic Reference Frame. Position paper adopted by the IAG Executive Committee, IAG Newsletter, Abril 2016, pp. 3-6.

INSTITUTO BRASILEIRO DE GEOGRAFIA E ESTATÍSTICA (IBGE). 2018. Relatório do Reajustamento da Rede Altimétrica com Números Geopotenciais 2018. Disponível em: <ftp://geoftp.ibge.gov.br/informacoes_sobre_posicionamento_geodesico/rede__ altimetrica/relatorio /relatorio_REALT_2018.pdf >. Acesso em: fev. 2020.

INTERNATIONAL EARTH ROTATION AND REFERENCE SYSTEMS SERVICE (IERS). Disponível em: https://www.iers.org/IERS/EN/Home/home_node.html. Acesso em: mar. 2020.

IHDE, J.; SÁNCHEZ, L.; BARZAGHI, R.; DREWES, H.; FOERSTE, C.; GRUBER, T.; LIEBSCH, G.; MARTI, U.; PAIL, R.; SIDERIS, M. Definition and proposed realization of the International Height Reference System (IHRS). Surveys in Geophysics, 2017, v. 38, n. 3, p. 549-570. 38(3), 549-570, 2017. DOI 10.1007/s10712-017-9409-3.

JESUS, R. M. L.; DE FREITAS, S. R. C. Potencialidade do Uso de Dados Geofísicos Aerolevantados para Aplicações Geodésicas. Revista Brasileira de Cartografia, 2020, v. 72, n. 1, p. 19-33. DOI. 10.14393/rbcv72n1-50760.

LEMOINE, F.G.; KENYON, S.C.; FACTOR, J.K.; TRIMMER, R.G.; PAVLIS, N.K.; CHINN, D.S.; COX, C.M.; KLOSKO, S.M.; LUTHCKE, S.B.; TORRENCE, M.H.; WANG, Y.M.; WILLIAMSON, R.G.; PAVLIS, E.C.; RAPP, R.H.; OLSON, T.R. The Development of the Joint NASA GSFC and the National IMagery and Mapping Agency (NIMA) Geopotential Model EGM96. GSFC Goddard Space Flight Center; 1998.

LEHMANN, R. Altimetry-gravimetry problems with free vertical datum. Journal of Geodesy, 2000, v. 74, n. 3-4, p. 327-334. DOI. 10.1007/s001900050290.

DE MATOS, A. C. O. C.; BLITZKOW, D.; MACHADO, W. C.; NUNES, M. A.; LENGRUBER, N. V.; XAVIER, E. M. L.; FORTES, L. P. S. MAPGEO2015: O novo modelo de ondulação geoidal do Brasil, Revista Brasileira de Cartografia, 2016, v. 68, n. 10.

MELO, L. F. S.; DE FREITAS, S. Avaliação de modelos globais do geopotencial para os estados do Maranhão e Piaú́. Boletim de Ciências Geodésicas, v. 18, n. 2, p. 203-224, 2012. DOI. 10.1590/S198221702012000200003.

NICACIO, E.; DALAZOANA, R.; DE FREITAS, S. R. C. Evaluation of recent combined global geopotential models in Brazil. Journal of Geodetic Science, 2018, v. 8, n. 1, p. 72-82. DOI. 10.1515/jogs-2018-0008.

PAVLIS, N.K.; HOLMES, S.A.; KENYON, S.C.; FACTOR, J.K. An Earth Gravitational Model to Degree 2160: EGM2008. EGU General Assembly 2008, Vienna, Austria, 13-18 Abril, 2008.

RENGANATHAN, V. Arctic sea ice freeboard heights from satellite altimetry, v. 71, n. 08. 2010. Tese 
Doutorado - Departamento de Engenharia em Geomática, Universidade de Calgary, Alberta, 2010.

SÁNCHEZ, L. Working Group on the Strategy for the Realization of the International Height Reference System (IHRS). Splinter Meeting of the WG on Strategy for the IHRS Realization, GGHS2016. Thessaloniki - Grécia, 2016.

SANTACRUZ-JARAMILLO, A. S.; DE FREITAS, S. R. C.; LUZ, R. T. Physical Connection Between BVRF Segments Based on Leveling Associated with Gravimetry. Boletim de Ciências Geodésicas, 2019, v. 25, n. 1. DOI 10.1590/s1982-21702019000100006.

TENZER, R.; VATRT, V.; ABDALLA, A.; DAYOUB, N. Assessment of the LVD offsets for the normalorthometric heights and different permanent tide systems - a case study of New Zealand. Applied Geomatics, 2011, v. 3, n. 1, p. 1-8. DOI 10.1007/s12518-010-0038-5.

TORGE, W.; MÜLLER, J. Geodesy. Walter de Gruyter, 2012.

UNITED STATES GEOLOGICAL SURVEY (USGS). USGS EROS Archive - Digital Elevation - Shuttle Radar Topography Mission (SRTM) 1 Arc-Second Global, 2019. Disponível em: <https://www.usgs.gov/centers/eros/science/usgs-eros-archive-digital-elevation-shuttle-radartopography-mission-srtm-non?qt-science_center_objects=0\#qt-science_center_objects $>$. Acesso em: fev. 2020.

UNITED NATIONS (UN). Resolution N. 69/266: A global geodetic reference frame for sustainable development. United Nation General Assembly, 2015. Disponível em: <https://undocs.org/en/A/RES/69/266>. Acesso em: fev. 2020.

YAMAZAKI, D.; IKESHIMA, D.; TAWATARI, R.; YAMAGUCHI, T.; O'LOUGHLIN, F.; NEAL, J. C.; SAMPSON, C.C.; KANAE, K.; BATES, P. D. A high-accuracy map of global terrain elevations. Geophysical Research Letters, 2017, v. 44, n. 11, p. 5844-5853. DOI. 10.1002/2017GL072874.

ZINGERLE, P.; PAIL, R.; GRUBER, T.; OIKONOMIDOU, X. The combined global gravity field model XGM2019e. Journal of Geodesy, 2020, v. 94, n. 7, p. 1-12. DOI. 10.1007/s00190-020-01398-0.

\title{
Biografia do autor principal
}

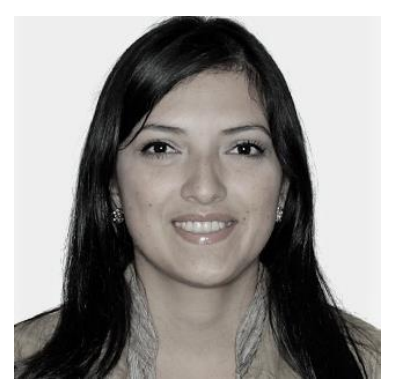

\begin{abstract}
Andrea Galudht Santacruz Jaramillo, nasceu no Equador em 1985. Engenheira Geógrafa e do Meio Ambiente pela Universidade das Forças Armadas (ESPE) em Quito - Equador (2010). Mestre em Ciências Geodésicas pelo Programa de PósGraduação em Ciências Geodésicas (PPGCG) da UFPR (2015). Bolsista de mestrado e doutorado pelo SENESCYT- Equador. Atualmente encontra-se culminando o doutorado no PPGCG. Membro do Laboratório de Referenciais Geodésicos e Altimetria por Satélites (LARAS). Membro do GT-III SIRGAS (Datum Vertical). Atua nas áreas: Geodesia Física, Gravimetria, Sistemas Geodésicos de Referência com ênfase no Sistema e Rede de Referência Internacional de Altitudes (IHRS/IHRF).
\end{abstract}

